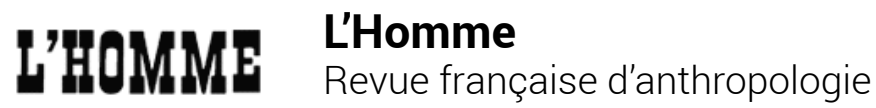

156 | octobre-décembre 2000

Intellectuels en diaspora et théories nomades

\section{Arthur T. Hatto, The Mohave Heroic Epic of Inyo- kutavere. Re-appraised and further interpreted on the basis of the edition of A. L. Kroeber and consultation of his field record}

Helsinki, Suomalainen Tiedeakatemia, Academia Scientiarum Fennica, 1999, 162 p., bibl. («FF Communication » 269)

\section{Claude Lévi-Strauss}

\section{(2) OpenEdition}

Édition électronique

URL : http://journals.openedition.org/lhomme/2757

DOI : 10.4000/lhomme.2757

ISSN : 1953-8103

Éditeur

Éditions de l'EHESS

Édition imprimée

Date de publication : 1 janvier 2000

Pagination : 285-287

ISBN : 2-7132-1348-7

ISSN : 0439-4216

\section{Référence électronique}

Claude Lévi-Strauss, « Arthur T. Hatto, The Mohave Heroic Epic of Inyo-kutavêre. Re-appraised and further interpreted on the basis of the edition of A. L. Kroeber and consultation of his field record ", L'Homme [En ligne], 156 | octobre-décembre 2000, mis en ligne le 29 novembre 2006, consulté le 24 septembre 2020. URL : http://journals.openedition.org//homme/2757 ; DOI : https://doi.org/10.4000/lhomme. 2757

Ce document a été généré automatiquement le 24 septembre 2020.

(c) École des hautes études en sciences sociales 


\section{Arthur T. Hatto, The Mohave Heroic Epic of Inyo-kutavêre. Re-appraised and further interpreted on the basis of the edition of A. L. Kroeber and consultation of his field record}

Helsinki, Suomalainen Tiedeakatemia, Academia Scientiarum Fennica, 1999, 162 p., bibl. («FF Communication » 269)

\section{Claude Lévi-Strauss}

$1 \quad$ EN 1902, Kroeber recueillit de la bouche d'un vieil Indien Mohave un récit épique qu'un auxiliaire indien traduisait en anglais au fur et à mesure. Il le publia dans cette langue en 1951 (A Mohave historical epic, University of California Anthropological Records 11,2).

Cette publication rencontra peu d'échos. Devereux s'y réfère brièvement à deux reprises dans son livre Mohave Ethnopsychiatry (1958), et le volume $10 \mathrm{du}$ Handbook of North American Indians (1983), au chapitre " Mohave ", la passe sous silence. Elle n'est même pas citée dans la bibliographie.

3 Il est vrai que sans se contenter d'un traitement purement ethnographique, Kroeber se livrait à des considérations variées sur les qualités littéraires du récit, la psychologie et les motivations romanesques des héros, de leurs adversaires, de personnages mineurs des deux sexes. Il comparait cette épopée avec les plus célèbres, Iliade et Mahâbhârata. Non sans gêne, G. H. Fathauer, autre spécialiste des Mohave et auteur de l'unique compte rendu paru de l'essai de Kroeber, y voyait un retour à « la tradition des humanistes " tout en reconnaissant qu'on devait à l'auteur des aperçus pleins de sensibilité (sympathetic) sur la culture et la personnalité mohave (American Anthropologist, 1952, 54, 4 : 549).

C'est, au contraire, de n'être pas allé assez loin que Arthur F. Hatto fait grief à Kroeber, dans un commentaire minutieux publié presque un siècle après que celui-ci eut 
découvert une œuvre épique et héroïque d'autant plus précieuse, souligne Hatto, qu'on n'en possède pas d'autre provenant des Amérindiens.

5 «Fort des quarante et quelques années de recherches sur la nature de la poésie épique orale qui manquèrent à Kroeber » (pp. 156-157), Hatto s'estime en droit de lui faire la leçon. Tout au long de son étude (je cite en anglais pour qu'on ne croie pas que je déforme), il lui reproche son « un-wonted looseness »; ou d'être, confronté à tel ou tel problème, « at a loss for an answer ». À l'occasion, Kroeber "violates the text » ou bien il a "rashly overshot the mark », etc. Les compliments qu'il lui adresse aussi ne sont pas dénués de perfidie. Ainsi Hatto déclare que sa propre interprétation, opposée à celle de Kroeber, "seems to us so easy and natural that only a great scholar could question it » (p. 159). Le narrateur lui-même n'a pas toujours compris le sens de ce qu'il racontait (p. 61).

6 Sur quoi porte le désaccord ? Kroeber a commis la faute inexcusable d'intituler le récit de son informateur «A Mohave historical epic » alors que, pour Hatto, ces épithètes sont incompatibles. Un poème épique peut tout au plus se donner un air de vraisemblance, il est historiquement sans valeur. Pourquoi ? Parce que les événements qu'il raconte sont inventés à des fins psychologiques et morales.

7 L'épopée mohave raconte que les ancêtres quittèrent jadis leur vallée fertile par libre choix, errèrent longtemps dans des régions désertiques, et durent lutter avec des fortunes diverses pour reconquérir leur pays contre les étrangers qui l'avaient occupé. L'abandon initial, inconcevable d'un point de vue historique, reste inexpliqué.

8 Or, affirme Hatto, «à la réflexion, ce choix ne semble pas seulement raisonnable, il est inévitable. Si les Mohave n'avaient pas volontairement et librement choisi de quitter la Vallée, ils devraient y avoir été forcés». Cela désobligerait leur voisins proches ou éloignés. Les Chemehuevi, Yuman, Walapai, Havasupai, Maricopa goûteraient peu que les Mohave fassent d'eux les auteurs de leurs malheurs passés. "Ainsi, dans le code de l'épopée, le seul moyen par lequel l'attachement passionné des Mohave à leur Vallée pouvait trouver une expression adéquate était la fiction (en fait, la folie) qu'ils avaient eux-mêmes librement choisi de la quitter » (p. 23). J'ai tenu à citer un exemple des explications prodiguées par l'auteur. Comme celle-ci, elles sont parfois désarmantes.

C'est qu'aux yeux de Hatto, éminent spécialiste, la poésie épique et héroïque constitue un «méta-genre » identifiable dans le monde entier par des caractères distinctifs. Il se propose de démontrer que l'épopée mohave en fait partie. Pour y parvenir, il mobilise toutes les ressources de la méthode comparative. Non seulement l'Ancien Testament, l' Iliade, Hésiode, le Nibelungenlied, l'Edda, Beowulf, les récits héroïques gallois, irlandais, la Chanson de Roland, le Kalevala qui sont de la poésie post-orale - on ne les connaît que par l'écrit -, mais tous les poèmes épiques recueillis de la bouche même des bardes: Ostyak, Yakut, Kirghiz, Tungus; ou provenant d'Asie du Sud, du Centre et du Sud-Est, de l'Afrique centrale et occidentale. On vacille sous cette masse de références accumulées dans le souci principal d'attribuer aux héros mohave des motivations psychologiques et morales semblables à celles d'Achille dans l'Iliade ou de tel personnage ob-ougrien. Peu de créations dues à des grands romanciers firent l'objet d'une étude psychologique aussi fouillée que celle consacrée par Hatto au héros Hipahipa, salué (le Nibelungenlied à l'appui) « as one of the superlative characters of heroic epic poetry worldwide» (p. 70). Cet enthousiasme s'étend au poème entier, qui témoigne d'un rare respect pour la personne de l'ennemi, « "alpha" for ethos [that] sets the Mohave epic squarely above at least one famous medieval European post-oral epic, 
above most Central Asian epics, above the Siberian epics with the exception of the ObUgrian and the Xosûn epic [...] in this one particular» (p.133). Quant au style de l'épopée mohave (dont nous n'avons qu'une traduction anglaise faite impromptu par un Indien), Aristote aurait pu le louer (p. 75).

Il faut rendre un hommage à Hatto. Pour conduire sa démonstration, il a dépouillé avec un soin extrême la littérature ethnographique sur les Mohave et leurs voisins. En s'aidant au besoin de la philologie, il l'utilise pour étayer sa thèse quand elle rend les rapprochements plus nets, ou bien pour discuter les cas où l'épopée mohave semble ignorer ou contredire les critères assignés à la poésie épique. D’un épisode qui lui parait hors de propos, Hatto se débarrasse en le qualifiant de "opportunistic excrescence ", allusion à l'insubordination des jeunes gens apparue dans la société mohave après 1850 . Une description détaillée de la technique du scalp aurait été aussi introduite à l'intention de Kroeber, car « no narrator among his own people at a great telling would have bored them with such platitudes » (p. 36). Le motif du tertre funéraire abritant les cendres du défunt manque-t-il dans l'épopée mohave? C'est que, chez eux, les combats simulés tenaient lieu des jeux funéraires en honneur dans l'Eurasie antique (pp. 55-56). Le rôle attribué aux chefs dans l'épopée ne correspond pas aux données ethnographiques. Mais celles-ci décrivent des chefs bien installés dans la Vallée mohave et menant des attaques au-dehors, tandis que le poème les représente à la tête de bandes errantes, dans le désert. Ils ont donc des conduites appropriées à chaque situation : remplacer l'une par l'autre « would have been a romantic falsification out of keeping with the Mohave temperament » (p. 105). Les explications passe-partout n'ont jamais rebuté l'herméneutique.

11 En introduisant la critique littéraire dans l'anthropologie, Kroeber ouvrait une voie que d'autres, tel Dell Hymes, ont suivie avec succès depuis. On peut se demander si Hatto, servi par une érudition impressionnante et par un style enflammé, ne l'a pas démesurément élargie. Des critères étrangers à notre discipline incitent à isoler des genres, à dresser des cloisons étanches entre mythe, quasi-mythe, conte, histoire, épopée.

12 Ainsi Kroeber avait cru nécessaire d'exclure de l'épopée mohave tout le début où il est raconté comment le héros culturel Mastamho octroya aux Mohave leur Vallée, et donna à chaque clan un nom. Ce préambule qui relève de la mythologie n'avait pas sa place, selon lui, en tête d'un récit de caractère historique. Hatto le réintègre, mais sépare en revanche le genre épique et héroïque de tous les autres.

13 Rien ne peut être plus éloigné des leçons de Dumézil. Elles nous ont au contraire appris à mener nos explorations «sur tous les types d'œuvre que produit habituellement la pensée humaine » en suivant une démarche « qui ne refuse à aucun moment [...] aucun moyen de connaissance »; car ce sont les mêmes schèmes qui sont utilisés « tantôt dans la mythologie, tantôt dans l'épopée ou l'histoire » (Mythe et épopée, I : 17, 25).

14 Le diable qui tenta Kroeber a confié à Hatto, presque cinquante années plus tard, le soin de conduire le bal. Il s'est acquitté de cette mission avec une allégresse, une juvénilité qui ne laisseraient pas soupçonner son grand âge. Cela force déjà l'admiration. On fera cependant plutôt confiance à la solide expérience ethnographique de Leslie Spier, un des rares spécialistes qui se soient intéressés au travail de Kroeber et qui, portant un jugement d'ensemble sur l'épopée mohave, voit en elle "a largely mythological narrative ». On pourrait le démontrer sur maint exemple. Tout le reste - rien que le reste - est littérature. 


\section{AUTEUR}

CLAUDE LÉVI-STRAUSS

Collège de France, Paris. 